\title{
1 Title: Contribution of PSD-95 protein to reward location memory
}

2 Abbreviated title: Contribution of PSD-95 to reward location memory

3 Anna Cały*1, Małgorzata Alicja Śliwińska*1,2, Magdalena Ziółkowska ${ }^{1}$, Kacper Lukasiewicz ${ }^{1}$,

4 Roberto Pagano ${ }^{1}$ Agata Nowacka ${ }^{1}$, Malgorzata Borczyk ${ }^{1}$, and Kasia Radwanska ${ }^{1}$

$5 \quad *$ these authors contributed equally

$6{ }^{1}$ Laboratory of Molecular Basis of Behavior, the Nencki Institute of Experimental Biology of Polish

7 Academy of Sciences, ul. L. Pasteura 3, Warsaw 02-093, Poland.

$8{ }^{2}$ currently Laboratory of Imaging Tissue Structure and Function, the Nencki Institute of

9 Experimental Biology of Polish Academy of Sciences, ul. L. Pasteura 3, Warsaw 02-093, Poland.

10 Corresponding author: Kasia Radwanska, Ph.D., Laboratory of Molecular Basis of Behavior, the

Nencki Institute of Experimental Biology of Polish Academy of Sciences, ul. L. Pasteura 3, Warsaw

02-093, Poland; e-mail: k.radwanska@nencki.gov.pl; tel: +48501736942

Key words: CaMKII, PSD-95, spatial memory, CA1, IntelliCages.

\section{Number of pages:}

Number of figures, tables, multimedia and 3D models (separately)

Figures (3), tables (0), multimedia and 3D models (0), supplementary figures (3)

Number of words for Abstract, Introduction, and Discussion (separately)

Abstract (207), Introduction (330), Discussion (940)

\section{Acknowledgments, Funding and Disclosure}

This work was supported by a National Science Centre (Poland) (Grant No. 2013/08/W/NZ4/00861 and 2015/19/B/NZ4/02996) to KR. AC, MŚ and KR designed the experiments; AC, MŚ, AN, MB, $\mathrm{K}$, RP, MŻ and KR performed and analysed the experiments; AC and KR wrote the manuscript. Authors report no financial interests or conflicts of interest. 


\section{Abstract}

The molecular mechanisms involved in formation of memory are still poorly understood. We focus here on the function of post-synaptic density protein 95 (PSD-95) and its phosphorylation by CaMKII in spontaneous learning about reward location in female mice. We show that formation of reward location memory leads to downregulation of PSD-95 protein in dendritic spines of the stratum radiatum, area CA1, and selective shrinkage of dendritic spines that contain PSD-95. ShRNA-driven, long-term downregulation of PSD-95 in the area CA1 decreases precision of memory. Autophosphorylation deficient CaMKII mutant mice (CaMKII:T286A) need more time than wild-type animals to learn the location of reward. The same impairment is observed after CA1targeted overexpression of CaMKII phosphorylation-deficient form of PSD-95 (PSD-95:S73A). In contrast to young adult mice, in aged animals reward location learning affects only spines that lack PSD-95. The frequency and size of the spines without PSD-95 are increased, while shRNA targeted to PSD-95 affects neither speed of learning nor precision of memory indicating alternative mechanisms to support successful memory formation in old mice. Altogether, our data suggest that dynamic regulation of PSD-95 expression is a mechanism that accelerates learning and improves precision of reward location memory in young mice. The function of PSD-95 in memory processes changes in aged animals. 


\section{Introduction}

The ability to find food and other natural rewards, as well as remember their location, is a key to animal survival. In humans, the importance of this process can be appreciated when perception of reward is aberrant, leading to exaggerated and inflexible reward seeking in drug addiction. Therefore, understanding molecular and cellular basis of reward seeking and memory of its location is crucial to understand processes involved in affective disorder such as addiction or depression.

Formation and consolidation of memory involves functional and structural plasticity of excitatory synapses ${ }^{1-3}$. Post-synaptic density protein 95 (PSD-95/SAP90), a member of the membrane-associated guanylate kinase (MAGUK) family, is highly abundant in the post-synaptic density (PSD) of an excitatory synapse and has been proposed to regulate different forms of synaptic transmission ${ }^{4-10}$, synapse structure and stability ${ }^{11-13}$ as well as formation and long-term stabilisation of memory ${ }^{14-17}$. PSD-95-dependent protein complexes interact both with AMPA- and NMDA-type glutamate receptors (AMPARs and NMDARs), and PSD-95 regulates NMDARdependent changes in AMPARs number ${ }^{18-21}$. Synaptic localization and function of PSD-95 is controlled by many interacting proteins and modifications, including phosphorylation, palmitoylation and ubiquitination ${ }^{9,22-27}$. In particular, upon stimulation of NMDAR, calcium and calmodulin-dependent kinase II (CaMKII)-driven phosphorylation of PSD-95 at serine 73 (PSD-95: S73) controls interactions of PSD-95 with NMDAR, synaptic localization of PSD-95, growth of dendritic spine and synaptic plasticity ${ }^{26,28}$. Still, the role of dynamic regulation of PSD-95 protein in the synapse is poorly understood in the context of memory process.

This study sought to understand the contribution of PSD-95 to reward location memory by integrating ex vivo analysis of PSD-95 expression, virally-mediated manipulation of PSD-95 expression and mobility, as well as behavioural analysis. We demonstrate that overexpression of CaMKII phosphorylation-deficient PSD-95 (PSD-95:S73A) in the area CA1 slows-down learning about reward location, while depletion of PSD-95 levels by shRNA impairs precision of memories. 
bioRxiv preprint doi: https://doi org/101101/590109; this version posted March 27, 2019. The copyright holder for this preprint (which was not certified by peer review) is the author/funder, who has granted bioRxiv a license to display the preprint in perpetuity. It is made available under aCC-BY-ND 4.0 International license.

69 This process operates in young mice, and is impaired in aged animals what may underlie age-related

70 cognitive decline.

71

72 


\section{Animals}

$\alpha$ CaMKII autophosphorylation-deficient mutant mice $\left(\alpha \mathrm{CaMKII-T286 \textrm {A } )}{ }^{29}\right.$, and heterozygous of Thy1-GFP M line mice (Thy1-GFP ${ }^{+-}$) ${ }^{30}$ were bred (as heterozygotes with the 129J/C57BL/6J background) in the Animal House of the Nencki Institute of Experimental Biology, and genotyped as previously described ${ }^{29,30}$. Young, adult mice were $5 \pm 1$ month-old during the behavioral training, whereas old individuals were $20 \pm 2$ month-old. Only female mice were used for all experiments, as male are too aggressive for group housing in the IntelliCages. All mice were housed with access to food and water ad libitum, and 12:12 hour dark-light cycle, $23-24^{\circ} \mathrm{C}$ and 35 $45 \%$ humidity. The studies were carried out in accordance with the European Communities Council Directive of 24 November 1986 (86/609/EEC), Animal Protection Act of Poland and approved by the 1st Local Ethics Committee in Warsaw. All efforts were made to minimize the number of animals used and their suffering.

\section{Reward location memory test in IntelliCages}

The IntelliCage system (NewBehavior AG, Zürich, Switzerland) (http://www.newbehavior.com/) consists of a large standard rat cage $(20.5 \mathrm{~cm}$ high, $40 \mathrm{~cm}$ x $58 \mathrm{~cm}$ at the top, $55 \mathrm{~cm} \times 37.5 \mathrm{~cm}$ at the base). In each corner, a triangular learning chamber is located with two bottles. To drink, only one mouse can go inside a plastic ring (outer ring: $50 \mathrm{~mm}$ diameter; inner ring: $30 \mathrm{~mm}$ diameter; $20 \mathrm{~mm}$ depth into outer ring) that ends with two $13 \mathrm{~mm}$ holes (one on the left, one on the right) that provides access to bottle nipples. Each visit to the corner, nosepoke at the doors governing access to the bottles, and lick were recorded by the system and ascribed to a particular animal. During experiments in each cage two corners were active. Groups of 8 to 15 mice were housed per cage.

Mice were subcutaneously injected with unique microtransponders which allow for mice identification in the IntelliCage (10.9 mm length, $1.6 \mathrm{~mm}$ diameter; Datamars, Slim Microchip TSL) under brief isoflurane anesthesia. Animals were allowed to recover for 3 days after the injection and after this time they were introduced to the IntelliCage. Experiments consisted of two phases: habituation (8-12 days) and learning. During habituation, animals had access to water in all corners. 
100 Four or five days before the learning phase, mice got access to 5\% sucrose solution (in tap water)

101 from the top of the cage to get familiarized with its taste. Baseline corner preference was measured

102 during the last day of the habituation as the $\%$ of visits or licks. During learning, water in less

103 preferred corner was replaced by $5 \%$ sucrose. The change in preference for the corner with sucrose

104 (\% visits performed to sucrose corner versus all visits) during training, compared with the

105 preference of the same corner during the baseline period $(\mathrm{H}-$ the last day of habituation) was used

106 as an index of spatial learning. All phases of the training were started at the beginning of the dark

107 phase (12:00 a.m.).

\section{Immunostaining on brain slices}

Mice were anesthetized and transcardially perfused with filtered PBS (Sigma-Aldrich) followed by 4\% PFA (Sigma-Aldrich) in PBS. Brains were removed and placed overnight in the same fixing solution and afterwards in $30 \%$ sucrose in PBS for three days. Next, coronal brain sections (40 $\mu \mathrm{m}$ thick) were prepared (Cryostat Leica CM1950, Leica Biosystems Nussloch GmbH, Wetzlar, Germany) and stored at $-20{ }^{\circ} \mathrm{C}$ in PBSAF [PBS, 15\% sucrose (Sigma-Aldrich), $30 \%$ ethylene glycol (Sigma-Aldrich), and $0.05 \% \mathrm{NaN}_{3}$ (SigmaAldrich)]. The sections were washed with PBS, PBS/0.3\%/Triton X-100 (Sigma-Aldrich) followed by 1-h incubation in a blocking solution (5\% normal donkey serum in PBS/0.3\% Triton X-100) and overnight incubation with the antibodies directed against PSD-95 (1:500, MAB1598; Merck-Millipore, RRID:AB_94278). Next, the sections were washed in PBS with $0.3 \%$ Triton X-100 and incubated for 90 minutes with the secondary antibody: anti-mouse Alexa Fluor 555 (1:500, A31570, Invitrogen, RRID:AB_2536180). The sections were mounted on glass microscope slides (Thermo Fisher Scientific), air-dried and coverslipped with Fluoromount-G medium with DAPI for fluorescence (00-4959-52, Invitrogen).

The staining was analyzed with the aid of confocal, laser-scanning microscope. Z-stacks of dendrites in the CA1 were acquired using Zeiss Spinning Disc microscope (63× oil objective and 1.66 digital magnification) (Zeiss, Göttingen, Germany). A series of 18 continuous optical sections $(67,72 \mu \mathrm{m} \times 67,72 \mu \mathrm{m})$, at $0.26 \mu \mathrm{m}$ intervals along the $z$-axis of the tissue section, were scanned. Six to eight Z-stacks of microphotographs were taken per animal, from every sixth section through 
the dorsal hippocampus (stratum radiatum of CA1 field) (one dendrite per neuron per image). Z-

stacks were reconstructed to maximal projections and analyzed with ImageJ software. Threshold tool was used, which identifies objects distinct from the background based on intensity. The density and average size of PSD-95+ puncta, as well as their co-localization were analyzed using Fiji software and measured using the analyze particle tool as previously described ${ }^{31}$. To analyze the images of the stained sections with overexpression of AAVs, a confocal microscope (magnification: x63, oil objective) (Leica TCS SP8, Leica Microsystems, Wetzlar, Germany) was used, and mean gray value of the microphotographs was assessed with ImageJ software.

Dendritic spines filled with GFP (in Thy1-GFP mice) were analyzed using semiautomatic SpineMagick! Software ${ }^{32}$. Data analysis was performed using scripts in Python. Overall we analyzed: 1112 spines from young, control mice; 2455 spines from young, learning mice; 972 spines from old, control animals and 1367 spines from old training group. Custom-written Python scripts were used for Fiji software to analyze co-localization of PSD-95+ puncta with dendritic spines.

\section{Stereotactic intracranial injections}

Mice were anaesthetized with isoflurane (5\% for induction, $1.5-2.0 \%$ after), fixed in the stereotactic frame (51503, Stoelting, Wood Dale, IL, USA), and their body temperatures were maintained using a heating pad. Stereotactic injections were performed bilaterally into CA1 region of hippocampus using coordinates from the Bregma: AP, $-2.1 \mathrm{~mm}$; ML, $\pm 1.1 \mathrm{~mm}$; DV, $-1.3 \mathrm{~mm}$ according to ${ }^{33} .0 .5 \mu$ l of virus solution was microinjected through beveled 26 gauge metal needle and $10 \mu 1$ microsyringe (SGE010RNS, WPI, USA) connected to a microsyringe pump (UMP3, WPI, Sarasota, USA), and its controller (Micro4, WPI, Sarasota, USA) at a rate $0.1 \mu 1 / \mathrm{min}$. The microsyringe was left in a place for additional 10 min following injection to prevent leakage of the vector. Mice were injected with AAV1/2 coding wild-type form of PSD-95 (AAV: $\alpha$ CaMKIIPSD95(WT)-mCherry-WPRE) $\left(0.5 \mu \mathrm{l} /\right.$ site, viral titer $\left.1,35 \times 10^{9} / \mu 1\right)$, the mutated form of PSD-95 with point substitution of serine 73 to alanine (AAV: $\alpha$ CaMKII-PSD95(S73A)-mCherry-WPRE) $\left(0.5 \mu \mathrm{l} /\right.$ site, viral titer $\left.9,12 \times 10^{9} / \mu \mathrm{l}\right)$, or control mCherry (AAV: $\alpha$ CaMKII-mCherry-WPRE $(0.5 \mu 1 /$ 
154 site, viral titer 7,5 x10 $/ \mu 1$, obtained from Deisseroth's Lab). Lentiviral vectors (LVs) coding short-

155 hairpin RNA silencing PSD-95 expression ( $\alpha$ CaMKII-shRNA(PSD95)-GFP $(0.5 \mu 1 /$ site, viral titer

$1562,52 \times 10^{8} / \mu 1$ ) (gift from Dr. Oliver M. Schlüter (European Neuroscience Institute Göttingen,

157 Germany) ${ }^{8}$ or control vector based on a pSUPER shRNA targeting the Renilla luciferase cloned

158 into pTRIP (H1-shRNA(luciferase)) $\left(0.5 \mu \mathrm{l} /\right.$ site, viral titer $\left.6,52 \times 10^{8} / \mu \mathrm{l}\right)$ (donated by Dr Katarzyna

159 Kalita, Nencki Institute of Experimental Biology, Warsaw, Poland) were used. The viruses were

160 prepared by Animal Model Core Facility at Nencki Institute.

After the surgery, animals were allowed to recover for 14 days before the training in the

Disc confocal microscope (magnification: 10x) was used to photograph the dorsal hippocampus and assess the extent of the viral expression.

\section{Statistical data analysis}

Data acquisition and quantification was performed in a group blind manner. All statistical analyses were performed using Prism 6 (GraphPad Software). The exact sample size (e.g., the number of mice or spines) of each experiment is provided in the relevant figures together with details of statistical tests. For behavioural data, immunostaining and dendritic spine analysis oneway and two-way analysis of variance (ANOVA), and post-hoc Tukey's multiple comparisons test were used. Dendritic spine volume did not follow normal distributions and were compared with Mann-Whitney test. For other parameters, unless specified, t-tests were performed. All data with normal distribution are presented as the means \pm standard error of the mean (SEM). For samples which did not follow normal distribution medians and interquartile range (IQR) are shown. The difference between the experimental groups was considered as significant if $\mathrm{p}<0.05$. 


\section{Results}

\section{Formation of memory about reward location downregulates PSD-95 protein in dendritic} spines.

To study neuronal processes underlying learning of reward location we used IntelliCage setup. In this setup the activity and spontaneous learning of female mice leaving in a group can be measured in close to ecologic conditions and without stressful intrusion of the experimentators ${ }^{34}$. We used young adult Thy1-GFP(M) mice $\left(5 \pm 1\right.$ month-old) ${ }^{30}$ (Fig. 1A) to analyse co-localisation of PSD-95 protein and dendritic spines as a proxy of training-induced synaptic remodelling ${ }^{35,36}$. Mice were trained to find sucrose reward in one of two active cage corners ${ }^{34}$ (Fig. 1B.i). Animals significantly increased preference of the rewarded corner during the first 30 minutes of the training, and continue to prefer this corner during the following 90 minutes (Fig. 1B.ii).

Next, we analysed dendritic spines in stratum radiatum of CA1 area (Fig. 1C.i), as this region is involve in formation of spatial memory ${ }^{37}$. Training did not affect density of spines (Fig. 1C.ii). However, median dendritic spines' areas were smaller after training as compared to control mice (Fig. 1C.iii), and distribution of dendritic spines' areas was shifted to smaller values after learning as compared to the spines analysed in control mice (Fig. 1C.iv).

To study the expression of PSD-95 protein we performed immunostaining with PSD-95specific antibody and analysed its co-localisation with dendritic spines. Intensity of PSD-95 immunostaining in the area CA1 was not changed in the learning mice as compared to the control group (Fig. 1D). When dendritic spines were segregated in two categories: with and without PSD95 [PSD-95(+) and (-)] (Fig. 1E.i), we observed that only 43\% of the spines contained PSD-95, which is very low as compared with previous studies showing that in the visual cortex (V1) over $80 \%$ of spines contained PSD-95 protein ${ }^{38}$. Therefore, to validate our method, we analysed PSD-95 protein expression in dendritic spines of the V1 cortex in young control animals. The frequency of dendritic spines with PSD-95 in V1 reached 80\%, as previously reported ${ }^{38}$. The frequency of PSD95-positive spines in the same animals in the area CA1 was $45 \%$. The spines in V1 were also bigger, and contained more PSD-95 puncta as compared to CA1 region (extended data Fig. 1-1). 
Thus, although we cannot exclude the possibility that we did not detect PSD-95 protein if it was expressed in very low quantity, we concluded that low frequency of dendritic spines that contain PSD-95 protein plausibly indicates low frequency of mature spines in the area CA1 ${ }^{39,40}$, as compared to the cortical region. in the control animals (Fig. 1E.ii). The analysis of the areas of spines showed that the spines with PSD-95 have higher median values than spines without PSD-95 (Fig. 1E.iii). Moreover, the median of PSD-95(+) spines' areas decreased after training while the median of PSD-95(-) spines' areas was not changed (Fig. 1E.iii). The change of PSD-95(+) spines was also observed as a shift of size distribution toward smaller values in learning mice as compared to the controls (Fig. 1E.vi). No analysed PSD-95 puncta to find that the total area of PSD-95 puncta per PSD-95(+) spines was decreased in the learning group, as compared with the controls (Fig. 1E.vi), while density of PSD95 puncta in the shaft increased (Fig. 1E.vii) suggesting translocation of the protein.

In summary, our data indicate remodelling of dendritic spines during memory formation that is dendritic spine type-specific. In young adult mice, training to locate sucrose reward results in shrinkage of big spines containing PSD-95 in the area CA1. At the same time PSD-95 protein level in dendritic spines is decreased and the protein is partly translocated to the shaft.

\section{PSD-95 regulates precision of reward location memory}

To test the function of PSD-95 protein in reward location memory, we used lentiviruses encoding short hairpin RNA (shRNA) targeted to PSD-95 mRNA (LV: $\alpha$ CaMKII-shRNA_PSD-95- 
compared with the control virus coding shRNA designed for Renilla luciferase (LV:H1shRNA_luciferase) (Fig. 2A.iii-iv). ShRNA for PSD-95 did not impair mice performance during initial 30 minutes of the training, however, later the preference of the reward corner of the mice transfected with shRNA for PSD-95 was lower than the preference of the control animals (Fig.

2AB.v).

In summary, our experiments indicate that long-term downregulation of PSD-95 protein in

CA1 does not affect formation of reward location memory but results in poor precision of memory, as demonstrated by long-term decrease of the reward corner preference. modifications. In particular, upon stimulation of NMDAR, calcium and calmodulin-dependent kinase II (CaMKII)-driven phosphorylation of PSD-95 at serine 73 (PSD-95: S73) controls interactions of PSD-95 with NMDAR, synaptic localization of PSD-95, growth of dendritic spine and synaptic plasticity 26,28 . We therefore decided to test whether CaMKII-dependent phosphorylation of PSD-95:S73 controls reward location memory.

First, to test the role of CaMKII in spatial memory formation, we used 4-month old, autophosphorylation-deficient $\alpha \mathrm{CaMKII}$ mutant mice ( $\alpha$ CaMKII-T286A) ${ }^{29}$. Autophosphorylation of CaMKII: threonine 286 decreases clustering of PSD-95 with NMDAR subunit, NR2B ${ }^{26}$. The young T286A mutants, as compared with the young WT mice, had decreased preference of the reward corner during training indicating impaired formation and precision of spatial memory (Fig. 2A).

To test the role of the interaction of CaMKII with PSD-95 protein in spatial memory 253 formation we used AAV1/2, coding wild-type (WT) and phosphorylation-deficient mutant PSD-95 254 protein at CaMKII-targeted Serine 73 (S73A) ${ }^{26,28}$. In control group we used AAV1/2 coding mCherry. The viruses were stereotactically injected into dorsal area CA1 of 4-month old, C57BL/6J 
mice (Fig. 2C.i), resulting in overexpression of PSD-95 protein (Fig. 2C.ii and iii). Two weeks after the surgery mice were trained (Fig. 2C.i). Overexpression of PSD-95(WT) did not affect the preference of the reward corner during training, as compared to mCherry control (Fig. 2C.v). Overexpression of phosphorylation-deficient mutant PSD-95(S73A), as compared to wild-type form of PSD-95, decreased preference of the reward corner during initial 30 minutes of training, but not preference of the reward corner in 10-visit bins (to make the number of learning trials equal between the experimental groups) to find similar effects as described for the timebins (extended data, Fig. 2-1). accelerates memory formation during initial 30 minutes of the training, while autophosphorylation of CaMKII is controls both speed and long-term precision of memory in young mice.

\section{Regulation of PSD-95 expression during reward location training is age dependent.}

A growing body of evidence indicates that during ageing many synaptic processes in the hippocampus are impaired (Burke and Barnes 2006, 2010), presumably leading to compromised precision of spatial memory and speed of learning (Hedden et al. 2004). We therefore asked whether learning-induced remodelling of PSD-95 protein at the synapse is altered in aged mice.

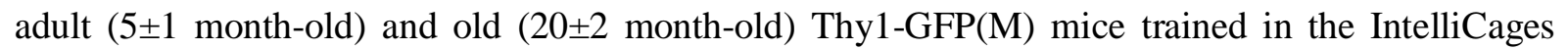
(Fig. 3A). Young mice were more active than old animals during the habituation, but not during the minutes of the training (Fig. 3A), up to circa 85\%, and there was no statistically significant 
10-visit bins (extended data, Fig. 3-1.A), suggesting no gross cognitive impairment in old Thy1GFP(M) mice.

Neither age nor training affected density of dendritic spines (Fig. 3B.i-ii). However, the medial of dendritic spines' areas in old control mice was significantly smaller spines than young controls (Fig. 3B.iii). Moreover, behavioural training resulted in increased median value of dendritic spines' areas in old animals (Fig. 3B.iii). These changes were also observed as shifts in distribution of values of dendritic spines' areas. Distribution of spines' areas of old control mice was shifted toward smaller values as compared to young control mice (Fig. 3B.iv). In old mice distribution of the spines' areas shifted toward larger values after training (Fig. 3B.v). as compared to the control young group, and it was not changed by the training (Fig. 3C).

Next, we calculated density and size of dendritic spines with and without PSD-95 protein.

As in young mouse, in old animals PSD-95(-) spines were more frequent than PSD-95(+) spines

(Fig. 3D.ii). However, in old mice density of PSD-95(-) spines increased after training over the lower in old mice as compared to the young animals (Fig. 3D.ii). The analysis of the areas of spines with and without PSD-95 protein showed that the spines with PSD-95 have higher median values than spines without PSD-95 (Fig. 3D.iii). Moreover, the median values of PSD-95(-) spines' areas increased after training while the median values of PSD-95(+) spines' areas did not change (Fig. 3D.iii). The change of PSD-95(-) spines in old mice was also observed as a shift of size distribution significant change in distribution of PSD-95(+) spines' areas was observed (Fig. 3D.iv). We also analysed PSD-95 puncta to find that the total area of PSD-95 puncta per spine in PSD-95(+) spines was lower in the old control mice, as compared to the young, control animals and it was not affected by the training (Fig. 3D.vi). The density of PSD-95 puncta in the shaft increased after training, both in young and old mice (Fig. 3D.vii). 
In summary, our data indicate age- and spine type-specific remodelling of dendritic spines

309

during memory formation. In young mice training resulted in shrinkage of big spines containing PSD-95. In old mice, the density of PSD-95(-) spines increased, suggesting removal of PSD-95 protein that was presumably translocated to the shaft. At the same time the average size of the spines without PSD-95 increased.

Next, to test the function of PSD-95 protein in old mice, we used lentiviruses encoding shRNA targeted to PSD-95 mRNA (LV: $\alpha$ CaMKII-shRNA_PSD-95-GFP) or luciferase ${ }^{8}$ (Fig. 2B). $20 \pm 1$ month old, C57BL/6J mice had LVs stereotactically injected into dorsal area CA1, to downregulate PSD-95 expression, and 14 days later they were trained in the IntelliCages (Fig. 3E.iii). ShRNA for PSD-95 did not impair mice performance neither during initial 30 minutes of the training, nor later (Fig. 3E.iii). Since downregulation of PSD-95 in CA1 of old mice increased activity of the mice (extended data, Fig. 3-1.B), we also analysed the preference of the reward corner in 10-visit bins (to make the number of learning trials equal between the experimental groups) to find similar effects as described for the time bins (extended data, Fig. 3-1.B). 


\section{Discussion}

327

328

329

330

In the current study we analyzed the molecular mechanisms of reward location memory. We show that formation of memory in young adult mice is accompanied by elimination of PSD-95 protein from large dendritic spines and dendritic spine shrinkage in the stratum radiatum of CA1 area. Using molecular manipulations in vivo we demonstrate that autophosphorylation of CaMKII and CaMKII-dependent destabilization of PSD-95 at the synapse by phosphorylation of PSD-95:serine 73 accelerates memory formation, as the speed of learning is compromised by overexpression of phosphorylation-deficient form of PSD-95:S73A. Long-term downregulation of PSD-95 decreases precision of reward location. In old mice, consolidation of reward location memory results in increased population of spines without PSD-95 and dendritic spines in this category grow. Overall, our data indicate that dynamic regulation of PSD-95 at the synapse is a mechanism for memory formation and stabilization that operates in young animals, but is impaired in aged mice. Thus in old age PSD-95-independent processes underlie learning.

We trained mice to find sucrose reward in one of two active corners of the IntelliCages ${ }^{34}$. The system allowed for on-line monitoring of mice performance during the training. Preference to visit reward corner was used as a measure of reward location memory and its precision. Both young and old mice increased preference of the rewarded corner during initial 30 minutes after reward location. In young mice the training resulted in shrinkage of dendritic spines that contained PSD-95 in stratum radiatum of the area CA1. The total number of the spines with PSD-95 was not altered, however, the size of PSD-95 clusters in spines was decreased. In contrast to young animals, reward location training in aged mice affected mostly spines without PSD-95. Their frequency and size were increased. The spines in old control mice where smaller than the spines in control young adults, however, after training they reached similar size. To our knowledge this is the first study that shows spine type- and age-specific downregulation of PSD-95 protein during memory processes. Since PSD-95 protein controls localization of AMPAR at the synapse ${ }^{19,20}$, AMPAR currents ${ }^{8,19,41}$, and synaptic plasticity ${ }^{9,10}$, the morphological and molecular changes we observe in young mice suggest that formation of memory about reward location is accompanied by weakening of CA1 
circuit, and this process is impaired in aged mice. This is in agreement with earlier findings showing

that formation of memory about spatial location of a novel object temporarily weakens synaptic transmission ${ }^{42,43}$. To fully validate whether synaptic transmission in CA1 is indeed altered in our model further experiments are needed. Currently, we can, however, conclude that PSD-95 scaffolding is disassembled during memory formation in young mice. To test the role of this process we performed virally-mediated local manipulations of PSD-95 expression.

CA1 area-targeted long-term downregulation of PSD-95 protein in young mice by overexpression of specific shRNA did not affect initial phase of learning, however, later it impaired precision of spatial memory. Young mice with depleted PSD-95 levels showed lower and less stable preference for the rewarded corner as compared with the control group. This finding is in agreement with the earlier studies showing that PSD-95 expression is dispensable for the formation and expression of recent contextual fear memories, but it is essential for their precision ${ }^{14,44}$. Surprisingly, this function of PSD-95 is impaired in aged mice which show similar precision of reward location memory to young mice, despite lower levels of PSD-95 protein in dendritic spines. The precision of reward location was also not affected in aged animals by further depletion of PSD95 in CA1 by PSD-95-targeted shRNA. Thus our data indicate that old mice use PSD-95independent, or possibly CA1-independent, strategy to precisely remember reward location. This hypotheses need, however, further validations.

Previously, it was shown that synaptic stimulation results in CaMKII-dependent mobilization of PSD-95 from dendritic spine ${ }^{28}$. This process relies on CaMKII-driven phosphorylation of PSD-95 on serine 73 and requires autophosphorylation of CaMKII:T286 ${ }^{26,28}$. The function of PSD-95:S73 phosphorylation in memory processes was never tested. Here we tested both the role of autophosphorylation of CaMKII:T286 and CaMKII-dependent phosphorylation of PSD-95:S73 in reward location memory. The training of autophosphorylationdeficient CaMKII mutant mice (T286A) and mice with local overexpression of phosphorylationdeficient PSD-95:S73A in the area CA1 indicate that these processes regulate and speed up early phase of learning. Moreover, autophosphorylation of CaMKII, but not phosphorylation of PSD- 
95:S73, is important for precision of reward location memory. Our data are in agreement with many

381

382

383

384 earlier studies showing that autophosphorylation of CaMKII, as a key regulator of synaptic plasticity ${ }^{29,45,46}$ and morphology of dendritic spines and PSDs ${ }^{47,48}$, also controls formation and flexibility of spatial and contextual memory ${ }^{29,48-50}$. We demonstrate, however, for the first time the role of CaMKII-dependent phosphorylation of PSD-95:S73 in memory.

Overall, our data show that in young animals learning about spatial location of reward induces elimination of PSD-95 protein from dendritic spines of CA1. Fast learning requires autophosphorylation of CaMKII and CaMKII-dependent phosphorylation of PSD-95 at serine 73. The precision of memory, but not the speed of learning, is sensitive to long-term downregulation of PSD-95 protein levels. Surprisingly in aged animals, this function of PSD-95 is not preserved, as depletion of PSD-95 does not affect precision of memory in old mice. We therefore conclude that in the aged animals, that have no signs of cognitive decline, alternative mechanisms support successful and precise memory formation. 


\section{References:}

396

397

398

399

400

401

402

403

404

405

406

407

408

409

410

411

412

413

414

415

416

417

418

419

1. Malenka, R. C. \& Bear, M. F. LTP and LTD: an embarrassment of riches. Neuron 44, 5-21 (2004).

2. Kessels, H. W. \& Malinow, R. Synaptic AMPA receptor plasticity and behavior. Neuron 61, 340-50 (2009).

3. Kemp, A. \& Manahan-vaughan, D. Hippocampal long-term depression $\square$ : master or minion in declarative memory processes $\square$ ? 30, (2007).

4. Ehrlich, I., Klein, M., Rumpel, S. \& Malinow, R. PSD-95 is required for activity-driven synapse stabilization. Proc. Natl. Acad. Sci. 104, 4176-4181 (2007).

5. El-Husseini, A., Schnell, E. \& Chetkovich, D. PSD-95 involvement in maturation of excitatory synapses. Science (80-. ). 290, 1364-8 (2000).

6. Chen, X. et al. Organization of the core structure of the postsynaptic density. Proc. Natl. Acad. Sci. 105, 4453-4458 (2008).

7. Elias, G. M., Elias, L. A. B., Apostolides, P. F., Kriegstein, A. R. \& Nicoll, R. A. Differential trafficking of AMPA and NMDA receptors by SAP102 and PSD-95 underlies synapse development. Proc. Natl. Acad. Sci. U. S. A. 105, 20953-20958 (2008).

8. Schlüter, O. M., Xu, W. \& Malenka, R. C. Alternative N-Terminal Domains of PSD-95 and SAP97 Govern Activity-Dependent Regulation of Synaptic AMPA Receptor Function. Neuron 51, 99-111 (2006).

9. $\mathrm{Xu}, \mathrm{W}$. et al. Molecular dissociation of the role of PSD-95 in regulating synaptic strength and LTD. Neuron 57, 248-62 (2008).

10. Stein, V., House, D. R. C., Bredt, D. S. \& Nicoll, R. A. Postsynaptic Density-95 Mimics and Occludes Hippocampal Long-Term Potentiation and Enhances Long-Term Depression. $J$. Neurosci. 23, 5503-5506 (2003).

11. Cane, M., Maco, B., Knott, G. \& Holtmaat, A. The Relationship between PSD-95 Clustering 
and Spine Stability In Vivo. J. Neurosci. 34, 2075-2086 (2014).

421

422

12. Chen, X. et al. PSD-95 Is Required to Sustain the Molecular Organization of the Postsynaptic Density. J. Neurosci. 31, 6329-6338 (2011).

13. Nikonenko, I. et al. PSD-95 promotes synaptogenesis and multiinnervated spine formation through nitric oxide signaling. J. Cell Biol. 183, 1115-27 (2008).

14. Fitzgerald, P. J. et al. Durable fear memories require PSD-95. Mol. Psychiatry 20, 901-912 (2015).

15. Komiyama, N. H. et al. SynGAP regulates ERK/MAPK signaling, synaptic plasticity, and learning in the complex with postsynaptic density 95 and NMDA receptor. J. Neurosci. 22, 9721-9732 (2002).

16. Elkobi, A., Ehrlich, I., Belelovsky, K., Barki-Harrington, L. \& Rosenblum, K. ERKdependent PSD-95 induction in the gustatory cortex is necessary for taste learning, but not retrieval. Nat. Neurosci. 11, 1149-51 (2008).

17. Migaud, M. et al. Enhanced long-term potentiation and impaired learning in mice with mutant postsynaptic density-95 protein. Nature 396, 433-439 (1998).

18. Lin, Y. Postsynaptic Density Protein-95 Regulates NMDA Channel Gating and Surface Expression. J. Neurosci. 24, 10138-10148 (2004).

19. Opazo, P., Sainlos, M. \& Choquet, D. Regulation of AMPA receptor surface diffusion by PSD-95 slots. Curr. Opin. Neurobiol. 22, 453-460 (2012).

20. Bats, C., Groc, L. \& Choquet, D. The Interaction between Stargazin and PSD-95 Regulates AMPA Receptor Surface Trafficking. Neuron 53, 719-734 (2007).

21. Nair, D. et al. Super-Resolution Imaging Reveals That AMPA Receptors Inside Synapses Are Dynamically Organized in Nanodomains Regulated by PSD95. J. Neurosci. 33, 1320413224 (2013). 
Surface Expression. Nuron 40, 595-607 (2003).

23. El-husseini, A. E. et al. Synaptic Strength Regulated by Palmitate Cycling on PSD-95 University of California at San Francisco. 108, 849-863 (2002).

24. Bianchetta, M. J., Lam, T. T., Jones, S. N. \& Morabito, M. A. Cyclin-Dependent Kinase 5 Regulates PSD-95 Ubiquitination in Neurons. J. Neurosci. 31, 12029-12035 (2011).

25. Wu, Q., Sun, M., Bernard, L. P. \& Zhang, H. Postsynaptic density 95 (PSD-95) serine 561 phosphorylation regulates a conformational switch and bidirectional dendritic spine structural plasticity. J. Biol. Chem. 292, 16150-16160 (2017).

26. Gardoni, F., Polli, F., Cattabeni, F. \& Luca, M. Di. Calcium - calmodulin-dependent protein kinase II phosphorylation modulates PSD-95 binding to NMDA receptors. 24, 2694-2704 (2006).

27. Craven, S. E. \& Bredt, D. S. Synaptic targeting of the postsynaptic density protein PSD-95 mediated by a tyrosine-based trafficking signal. J. Biol. Chem. 275, 20045-20051 (2000).

28. Steiner, P. et al. Destabilization of the postsynaptic density by PSD-95 serine 73 phosphorylation inhibits spine growth and synaptic plasticity. Neuron 60, 788-802 (2008).

29. Giese, K. P., Fedorov, N. B., Filipkowski, R. K. \& Silva, a J. Autophosphorylation at Thr286 of the alpha calcium-calmodulin kinase II in LTP and learning. Science 279, 870-873 (1998).

30. Feng, G. et al. Imaging neuronal subsets in transgenic mice expressing multiple spectral variants of GFP. Neuron 28, 41-51 (2000).

31. Fedulov, V. et al. Evidence that long-term potentiation occurs within individual hippocampal synapses during learning. J. Neurosci. 27, 8031-9 (2007).

32. Ruszczycki, B., Wlodarczyk, J. \& Kaczmarek, L. Method and a System for Processing an Image Comprising Dendritic Spines. (2014). 
Press, 2001).

471

34. Harda, Z. et al. Autophosphorylation of $\alpha \mathrm{CaMKII}$ affects social interactions in mice. Genes, Brain Behav. (2018). doi:10.1111/gbb.12457

35. Graziane, N. M. et al. Opposing mechanisms mediate morphine- and cocaine-induced generation of silent synapses. (2016). doi:10.1038/nn.4313

36. Matsuo, N., Reijmers, L. \& Mayford, M. Spine-type-specific recruitment of newly synthesized AMPA receptors with learning. Science 319, 1104-7 (2008).

37. Tsien, J. Z., Huerta, P. T. \& Tonegawa, S. The essential role of hippocampal CA1 NMDA receptor-dependent synaptic plasticity in spatial memory. Cell 87, 1327-1338 (1996).

38. Villa, K. L. et al. Inhibitory Synapses Are Repeatedly Assembled and Removed at Persistent Sites In Vivo. Neuron 89, 756-769 (2016).

39. Sans, N. et al. A Developmental Change in NMDA Receptor-Associated Proteins at Hippocampal Synapses. J. Neurosci. 20, 1260-1271 (2000).

40. Attardo, A., Fitzgerald, J. E. \& Schnitzer, M. J. Impermanence of dendritic spines in live adult CA1 hippocampus. Nature 523, 592-596 (2015).

41. Beique, J.-C. et al. Synapse-specific regulation of AMPA receptor function by PSD-95. Proc. Natl. Acad. Sci. 103, 19535-19540 (2006).

42. Kemp, A. \& Manahan-Vaughan, D. Hippocampal long-term depression and long-term potentiation encode different aspects of novelty acquisition. Proc. Natl. Acad. Sci. 101, 8192-8197 (2004).

43. Goh, J. J. \& Manahan-Vaughan, D. Spatial object recognition enables endogenous LTD that curtails LTP in the mouse hippocampus. Cereb. Cortex 23, 1118-1125 (2013).

44. Nagura, H. et al. Impaired synaptic clustering of postsynaptic density proteins and altered signal transmission in hippocampal neurons, and disrupted learning behavior in PDZ1 and PDZ2 ligand binding-deficient PSD-95 knockin mice. Mol. Brain 5, 43 (2012). 
45. Coultrap, S. J. et al. Autonomous CaMKII mediates both LTP and LTD using a mechanism for differential substrate site selection. Cell Rep. 6, 431-7 (2014).

46. Ohno, M., Sametsky, E. A., Silva, A. J. \& Disterhoft, J. F. Differential effects of $\alpha$ CaMKII mutation on hippocampal learning and changes in intrinsic neuronal excitability. Eur. $J$. Neurosci. 23, 2235-2240 (2006).

47. Okamoto, K., Narayanan, R., Lee, S. H., Murata, K. \& Hayashi, Y. The role of CaMKII as an F-actin-bundling protein crucial for maintenance of dendritic spine structure. Proc Natl Acad Sci U S A 104, 6418-6423 (2007).

48. Radwanska, K. et al. Mechanism for long-term memory formation when synaptic strengthening is impaired. Proc. Natl. Acad. Sci. U. S. A. 108, 18471-5 (2011).

49. Irvine, E. E., von Hertzen, L. S. J., Plattner, F. \& Giese, K. P. alphaCaMKII autophosphorylation: a fast track to memory. Trends Neurosci. 29, 459-65 (2006).

50. Irvine, E. E., Vernon, J. \& Giese, K. P. AlphaCaMKII autophosphorylation contributes to rapid learning but is not necessary for memory. Nat. Neurosci. 8, 411-412 (2005). 


\section{$513 \quad$ Figures}

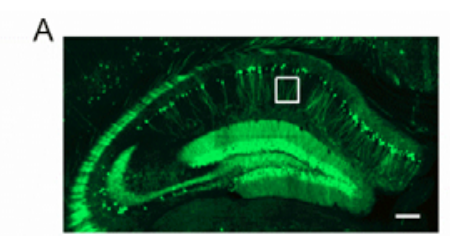

C (i) Control Learning

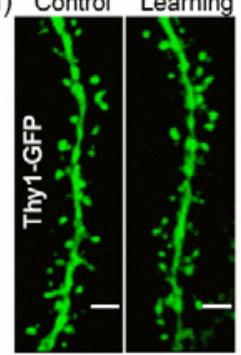

D (i) Control Learning

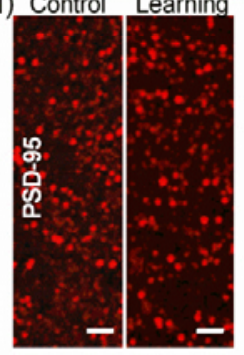

(iv)

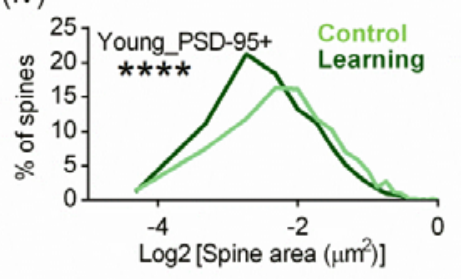

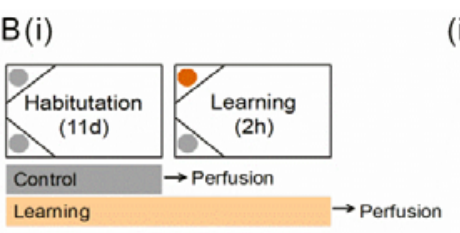

(iii)

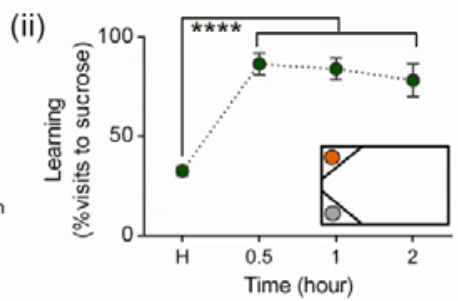

(iv)
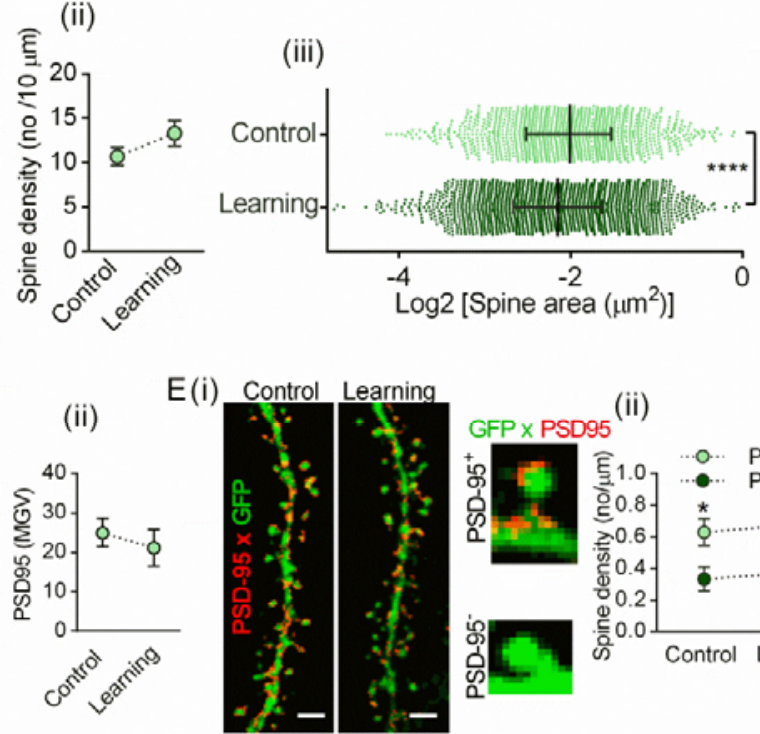

(v)

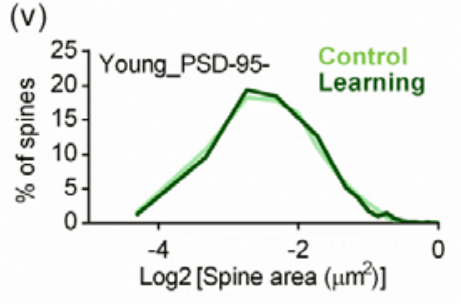

(ii)
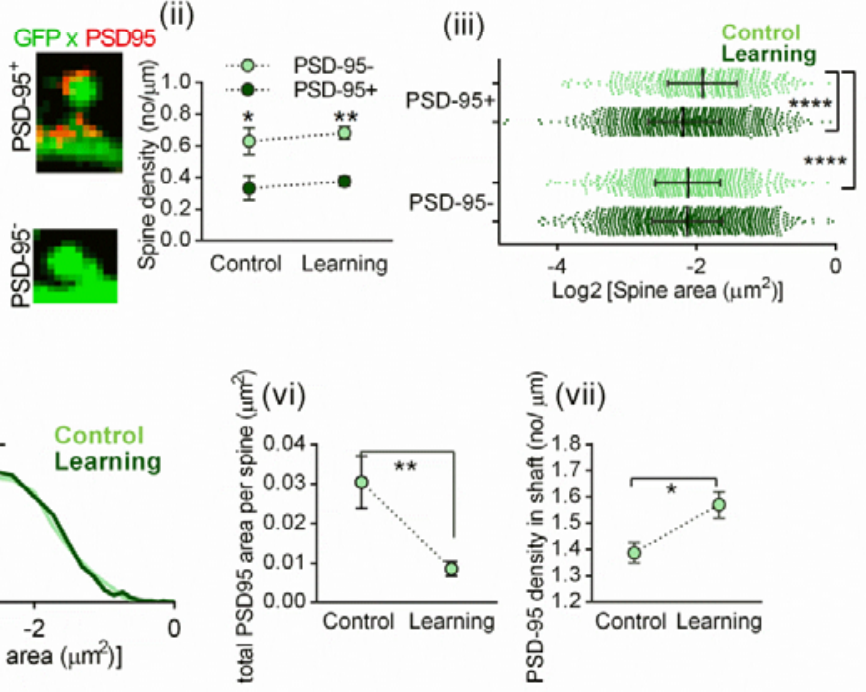

514

515 Figure 1. Learning-induced remodeling of PSD-95 protein scaffolding and dendritic spines.

516 (A) Representative image of dorsal hippocampus of Thy1-GFP(M) mouse. A white rectangle

517 indicates the area (stratum radiatum of dorsal area CA1) were dendritic spines and protein

518 expression were analyzed. Scale bar: $200 \mu \mathrm{m}$.

519 (B) (i) Cage setups during training and experimental timeline. The cages had two active corners

520 during training: with water (gray circle) and sucrose (orange circle). (ii) Mean+/- SEM preference

521 of the reward corner during training (control mice, $\mathrm{n}=5$; learning, $\mathrm{n}=6$ ). Mice increased

522 preference of the reward corner during the first 30 minutes of the training $(* * * * \mathrm{p}<0.0001, \mathrm{RM}$ 
ANOVA with Tukey's multiple comparisons test). H, preference of the corner during the last day of the habituation. Inset, cage setup during training.

(C) (i) Representative high resolution images of dendritic fragments of the control and trained Thy1-GFP(M) mice, scale bars: $2 \mu \mathrm{m}$. (ii) Linear density of dendritic spines was not affected by the training (t-test, $\mathrm{t}(9)=1.447, \mathrm{p}>0.05)$. (iii) Dendritic spines shrank during training $(* * * * * \mathrm{p}<$ spines $=4 / 1112$; learning, mice/ spines $=5 / 2455$ ) with medians and interquartile range. Scales are the spine sizes in learning mice shifted from control to smaller sizes $(* * * p=0.0004$, KolmogorovSmirnov test, $\mathrm{D}=0.07417)$.

(D) (i) Representative high resolution images of PSD-95 immunostaining in control and trained mice, scale bars: $2 \mu \mathrm{m}$. (ii) Mean gray value of the images was not affected by the training (p > 0.05 , t-test, $\mathrm{t}(9)=0.800 ;$ control, $\mathrm{n}=5$ and trained mice, $\mathrm{n}=6$ ).

(E) (i) Representative high resolution images of PSD-95 immunostaining co-localized with dendritic fragments of the control and trained Thy1-GFP(M) mice, scale bars: $2 \mu \mathrm{m}$. Right, spines with PSD-95 (PSD-95+) and without PSD-95 (PSD-95-). (ii) Average+/-SEM density of the spines. Spines without PSD-95 are more frequent than spines with PSD-95. Training does not affect density

540 of the spines $(* \mathrm{p}<0.05, * * \mathrm{p}<0.001$ PSD-95- vs PSD-95+, two-way ANOVA with Tukey's multiple comparisons test) (control PSD-95-, mice/ spines = 4/ 613; learning PSD-95-, mice/ spines = 5/ 1430; control PSD-95+, mice/ spines = 4/ 440; learning PSD-95+, mice/ spines = 5/ 1020). (iii) In control mice PSD-95+ spines are bigger than PSD-95- spines $(* * * * p<0.0001)$. Only PSD-95+ spines shrank after training $(* * * * p<0.0001$, Kruskal-Wallis ANOVA followed by Dunn's multiple comparisons tests, $\mathrm{H}=35.18$ ). (iv) Distribution of PSD-95+ spines' areas shifted from control to

546 smaller values after training $(* * * * p<0.0001$, Kolmogorov-Smirnov test, $\mathrm{D}=0.1501)$. (v) The

547 distribution of PSD-95- spines' areas did not change after learning ( $\mathrm{D}=0.02615)$. (vi) Average area 
bioRxiv preprint doi: https://doi org/10.1101/590109; this version posted March 27, 2019. The copyright holder for this preprint (which was not certified by peer review) is the author/funder, who has granted bioRxiv a license to display the preprint in perpetuity. It is made available under aCC-BY-ND 4.0 International license.

$549(* * \mathrm{p}<0.01, \mathrm{t}$-test, $\mathrm{t}(8)=3.826)$. (vii) Average density of PSD-95 puncta in dendritic shaft

550 increased after training (control, $\mathrm{n}=4$; learning $=6)(* \mathrm{p}<0.05$, t-test, $\mathrm{t}(8)=2.927)$. 


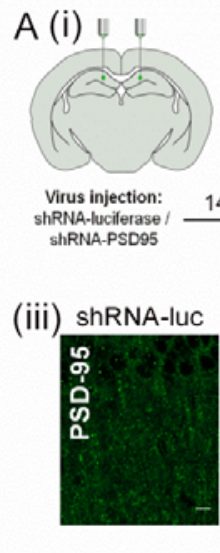

B (i)

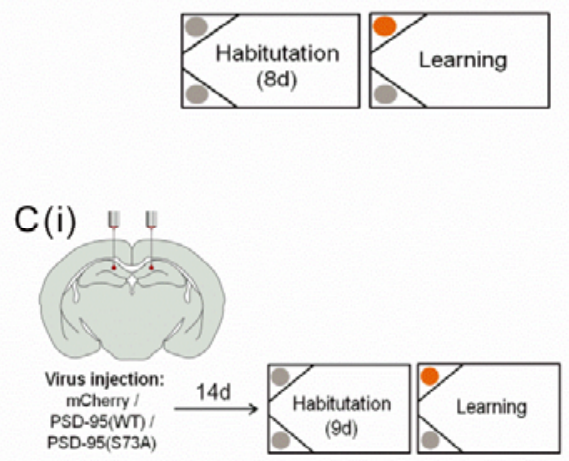

(iii)

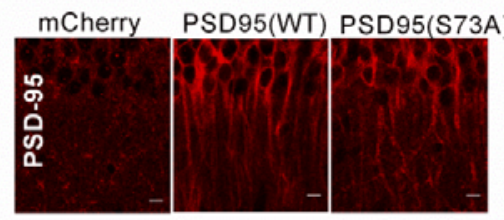

(iv)

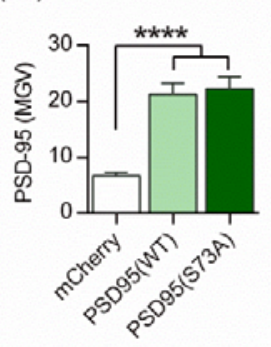

(v) (ii)

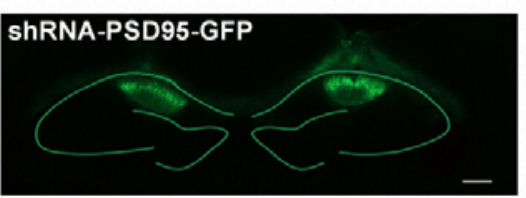

(v)

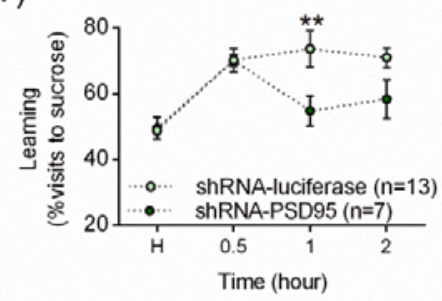

(ii)

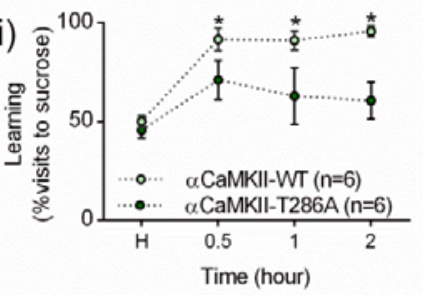

(ii) mCherry

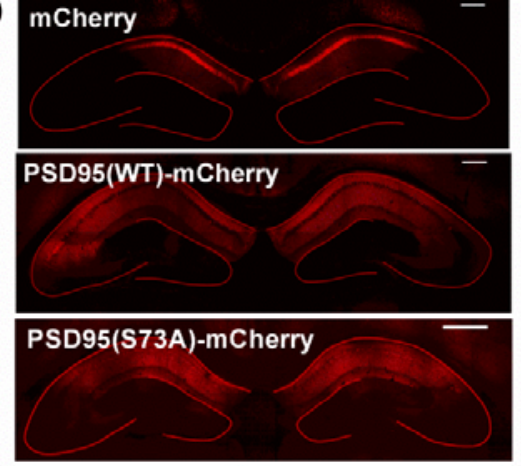

(vi)

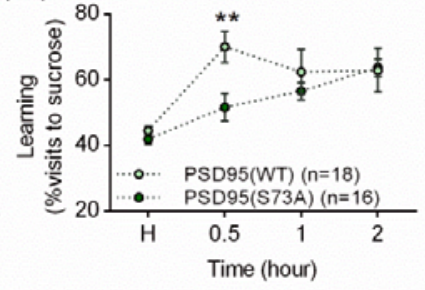

Figure 2. PSD-95 protein in the area CA1 controls spatial learning.

554 (A) (i) Experimental timeline. (ii) Representative microphotography of dorsal hippocampus with

555 local expression of lentiviruses coding shRNA targeted to PSD-95 (LV:aCaMKII_shRNA PSD-

556 95_GFP). Scale bar: $100 \mu \mathrm{m}$. (iii) Microphotographs of PSD-95 immunostaining in the stratum

557 radiatum of dorsal area CA1 after local expression of shRNA to Renilla luciferase 
559

560

561

562

563

564

565

566

567

568

569

570

571

572

573

574

575

576

577

578

579

580

$10 \mu \mathrm{m}$. (iv) shRNA for PSD-95 decreased mean gray value of PSD-95 immunostaining $(* * \mathrm{p}=$ 0.0054, t-test, $\mathrm{t}(6)=4.243$; mice with shRNA-luc: $\mathrm{n}=5$; shRNA-PSD95: $\mathrm{n}=3$ ). (v) ShRNA for PSD-95 impaired preference for reward corner after 1 and 2 hours of the training $(* * p<0.01$, Twoway RM ANOVA with Sidak's multiple comparisons test, virus: $F_{1,76}=12.72, p=0.0006$, time: $F_{7}$, $\left.{ }_{76}=8.611, \mathrm{p}<0.0001\right) . \mathrm{H}$, preference of the corner during the last day of the habituation.

(B) (i) Experimental timeline of spatial training of $\alpha \mathrm{CaMKII}-\mathrm{T} 286 \mathrm{~A}$ mutant mice and their WT littermates. (ii) T286A mutants are impaired in formation of spatial memory $(* \mathrm{p}<0.5$, two-way ANOVA with Sidak's multiple comparisons test, genotype: $F_{1,72}=11.02, p=0.001$, time: $F_{7,72}=$ $5.173, \mathrm{p}<0.0001)$

(C) (i) Experimental timeline. (ii) Representative images showing bilateral expression of AAV1/2 coding PSD95(WT)_mCherry, PSD95(S73A)_mCherry or mCherry in the area CA1. Scale bars: $100 \mu \mathrm{m}$. (iii) Representative microphotographs of the area CA1 showing PSD-95 protein immunostaining after viral infection. Scale bars: $10 \mu \mathrm{m}$. (iv) Transfection of CA1 area with AAVs coding PSD95(WT)_mCherry, PSD95(S73A)_mCherry resulted in overexpression of PSD-95 protein $\left(* * * * \mathrm{p}<0.0001\right.$, one-way ANOVA by Tukey's multiple comparisons test, virus: $\mathrm{F}_{2,22}=$ 24.30, $\mathrm{p}<0.0001)$. (v) Overexpression of PSD-95(WT) did not affect preference for the reward corner (Two-way RM ANOVA with Sidak's multiple comparisons test, virus: $\mathrm{F}_{1,36}=0.5392, \mathrm{p}=$ 0.4675; time: $\mathrm{F}_{3,108}=12.53, \mathrm{p}<0.0001$ ), (vi) while overexpression of phosphorylation deficient PSD-95(S73A) decreased preference for the rewarded corner during the first $0.5 \mathrm{hr}$ of the training $\left(* * \mathrm{p}<0.01\right.$, Two-way RM ANOVA with Sidak's multiple comparisons test, virus: $\mathrm{F}_{1,30}=3.139, \mathrm{p}=$ 0.0866; time: $\left.\mathrm{F}_{3,90}=8.053, \mathrm{p}<0.0001\right)$. 

aCC-BY-ND 4.0 International license.

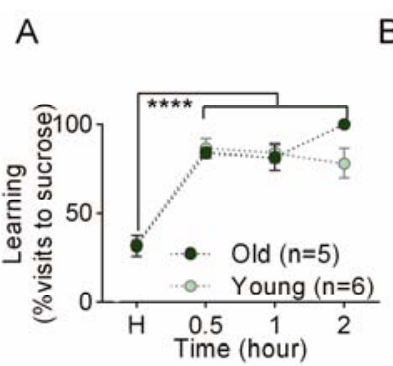

(iv)

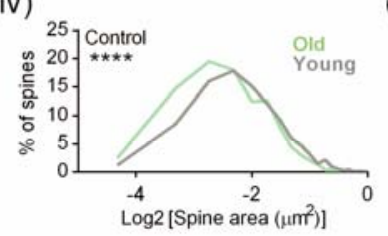

(ii)

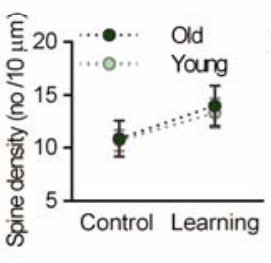

(iii)

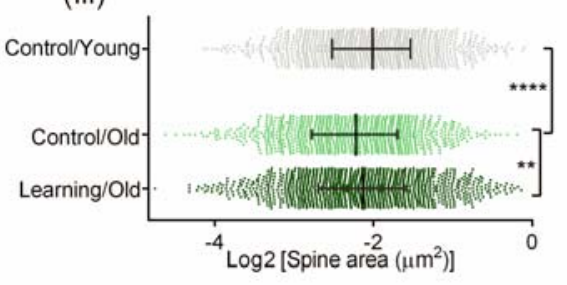

(ii) (v)

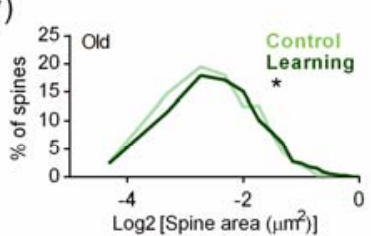

C(i) Control Learning

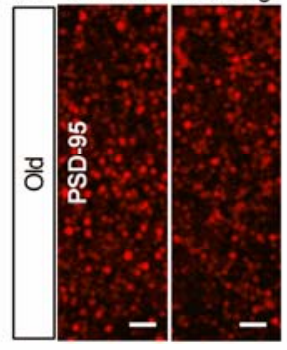

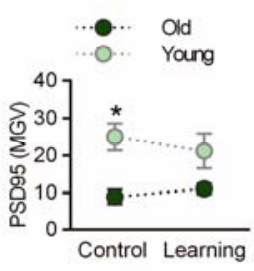

D (i) Control Learning

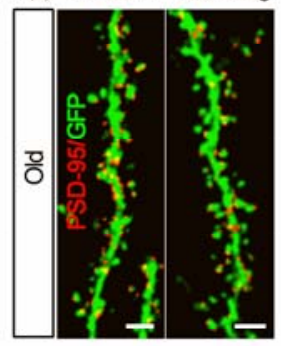

(ii) $\quad$ Old_PSD-95(-)
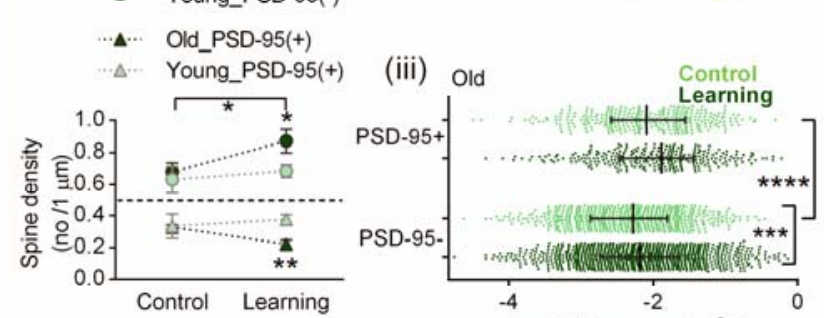

$\log 2$ [Spine area $\left(\mu \mathrm{m}^{2}\right)$ ]

(v)
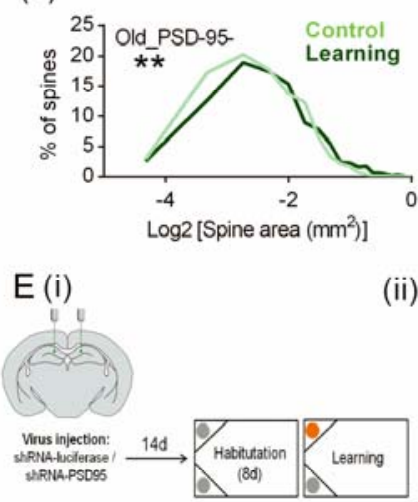

(vi)

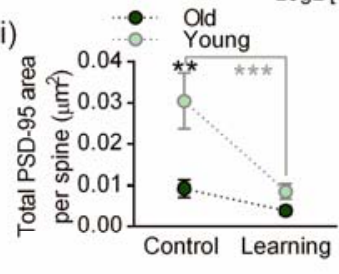

(vii)

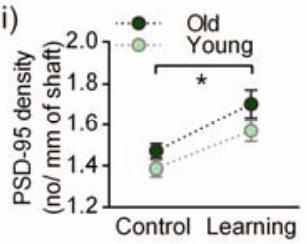

(iv)

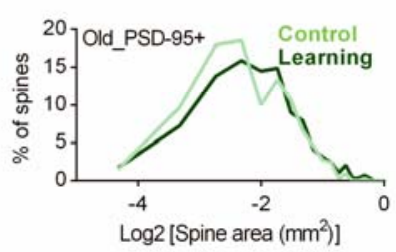

(iii)

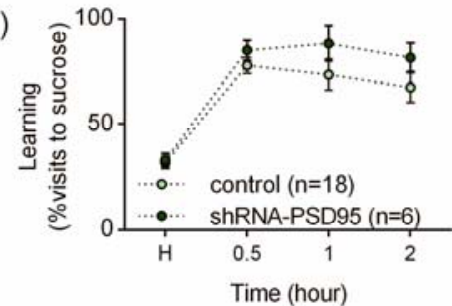

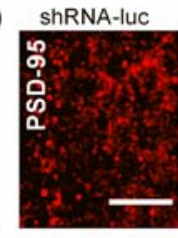

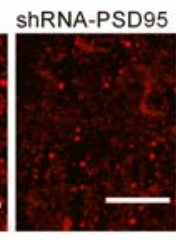

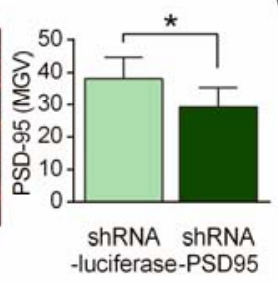

Time (hour)

Figure 3. Learning-induced remodeling of dendritic spines in old mice.

(A) Mean+/-SEM preference for the reward corner during training of old (20 \pm 1 month old) and young, adult (5 \pm 1 month old) Thy1-GFP(M) mice. Both for young and old mice, the preference of the reward corner was higher during training as compared to the habituation $(\mathrm{H})$. No difference in reward corner preference was observed between the old and young Thy1-GFP(M) mice $\left(* * * * \mathrm{p}<0.0001, \mathrm{RM}\right.$ ANOVA with Tukey's multiple comparisons test; time: $\mathrm{F}_{3,32}=51.88, \mathrm{p}<$ 0.0001; age: $F_{1,32}=1.037, p=0.3162$ ). All data for the young mice are the same as in Fig. 1 . 
(B) (i) Representative high resolution images of dendritic fragments of the old, control and trained

Thy1-GFP(M) mice, scale bars: $2 \mu \mathrm{m}$. (ii) Mean \pm SEM linear density of dendritic spines in old and

591

592

593

594

595 young mice. Linear density of dendritic spines was not affected by the training. There was no difference in spine density in old and young animals (old control mice, $\mathrm{n}=4$, old learning, $\mathrm{n}=5$, two-way ANOVA, age: $\mathrm{F}_{1,16}=0.077, \mathrm{p}=0.785$; training: $\mathrm{F}_{1,16}=3.401, \mathrm{p}=0.084$ ). (iii) Dendritic spines of old control mice were smaller than spines of young control mice, and old trained animals $(* * * * * \mathrm{p}<0.0001, * * \mathrm{p}<0.01$, Kruskal-Wallis test with Dunn's multiple comparisons test, $\mathrm{H}=40.76$, $\mathrm{p}<0.0001$ ). Graph shows data for individual spines (control, $\mathrm{n}=972$; learning, $\mathrm{n}=1327$ ) with medians and interquartile range. Scales are $\log _{2}$-tranformed. All statistical tests are performed on nontransformed data. (iv) Distribution of spines' areas of old control mice is shifted toward smaller values as compared to young control mice $(* * * * p<0.0001$, Kolmogorov-Smirnov test. (v) In old mice distribution of the spines' areas shifted toward larger values after training $(* \mathrm{p}=0.015$, Kolmogorov-Smirnov test).

(C) (i) Representative high resolution images of PSD-95 immunostaining in old, control and trained mice, scale bars: $2 \mu \mathrm{m}$. (ii) Mean gray value of images was lower in old control mice as compared to young control animals, and it did not differ from the old, trained mice $(* \mathrm{p}<0.05$, two-way ANOVA with Tukey's multiple comparisons test, age: $\mathrm{F}_{1,16}=13.59, \mathrm{p}=0.0020$; training: $\mathrm{F}_{1,16}=$ 0.0597, $\mathrm{p}=0.810$, interaction: $\left.\mathrm{F}_{1,16}=0.6769, \mathrm{p}=0.4227\right)$.

(D) (i) Representative high resolution images of PSD-95 immunostaining co-localized with dendritic fragments of the old, control and trained Thy1-GFP(M) mice, scale bars: $2 \mu \mathrm{m}$. (ii) Average $\pm+/-S E M$ density of the spines with (+) and without PSD-95 (-). Spines without PSD-95 are more frequent than spines with PSD-95. Training increased density of PSD-95(-) spines in old mice $\left({ }^{*} \mathrm{p}<0.05\right)$, and they have more PSD-95(-) spines than young trained mice $(* \mathrm{p}<0.05$, two-way ANOVA with Tukey's multiple comparisons test, training: $\mathrm{F}_{1,13}=3.532, \mathrm{p}=0.082$; age: $\mathrm{F}_{1,13}=$ 3.258, $\mathrm{p}=0.0943)$. After training old mice have less PSD-95(+) spines than young trained mice $\left(* \mathrm{p}<0.05\right.$, two-way ANOVA with Tukey's multiple comparisons test, training: $\mathrm{F}_{1,13}=0.7914, \mathrm{p}=$ 0.3898; age: $F_{1,13}=4.734, p=0.0486$ ). (iii) In the control group, PSD-95(+) spines are bigger than 
PSD-95(-) spines $(* * * * \mathrm{p}<0.0001)$. Only PSD-95(+) spines grew after training $(* * * * \mathrm{p}<0.0001$,

617 Kruskal-Wallis ANOVA followed by Dunn's multiple comparisons tests, $\mathrm{H}=50.62$ ) (control PSD-

95-, mice/ spines = 4/ 641; learning PSD-95-, mice/ spines = 5/ 1077; control PSD-95+, mice/

spines $=4 / 329$; learning PSD-95+, mice/ spines = 5/ 289). (iv) Distribution of PSD-95(+) spines'

areas of old mice did not change after training $(p>0.05$, Kolmogorov-Smirnov test, $D=0.094) .(v)$

621

The distribution of PSD-95(-) spines' areas shifted to bigger values after training $(* * \mathrm{p}<0.01$, training $\left(* * \mathrm{p}<0.01\right.$, two-way ANOVA with Tukey's multiple comparisons test, age: $\mathrm{F}_{1,13}=15.02, \mathrm{p}$ $625=0.0019$, training: $\mathrm{F}_{1,13}=14.34, \mathrm{p}=0.0023$ ). (iv) Average density of PSD-95 puncta in dendritic shaft increased after training both in young and old mice $\left({ }^{*} \mathrm{p}<0.05\right.$, two-way ANOVA with Tukey's multiple comparisons test, age: $\mathrm{F}_{1,16}=3.707, \mathrm{p}=0.0721$, training: $\left.\mathrm{F}_{1,16}=13.74, \mathrm{p}=0.0019\right)$. t-test, $\mathrm{t}(9)=2.292$ ), scale bars: $10 \mu \mathrm{m}$. (iii) CA1 area-targeted shRNA designed for PSD-95 did not

$632=0.1239$, time: $F_{3,69}=19.87, p<0.0001$, interaction: $\left.F_{3,69}=0.3756, p=0.7709\right) . H$, preference of 

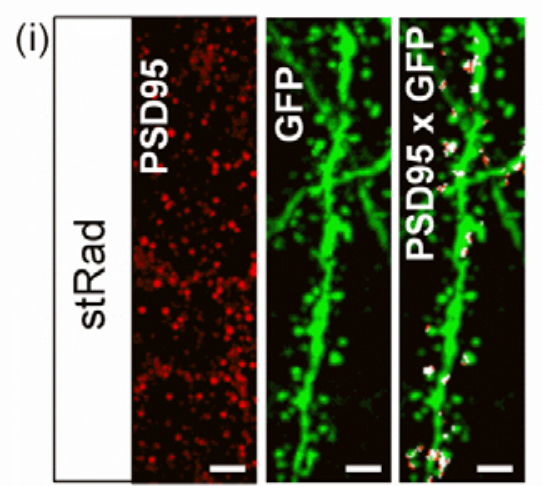

(iii)

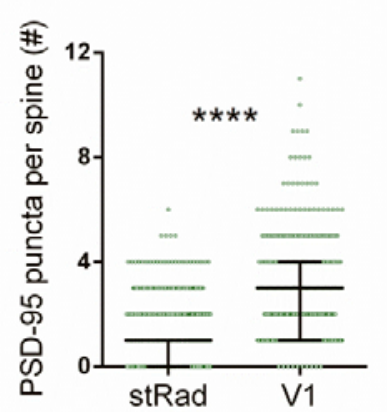

(ii)

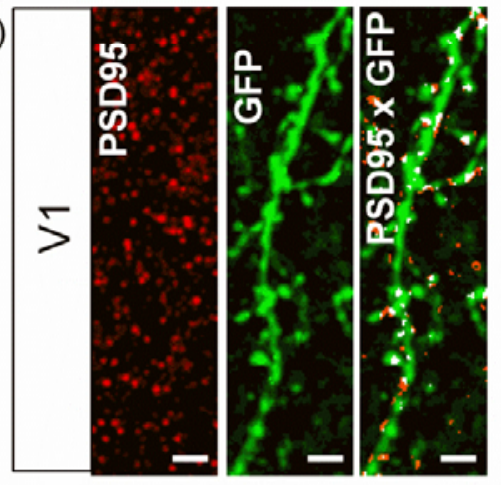

(v)

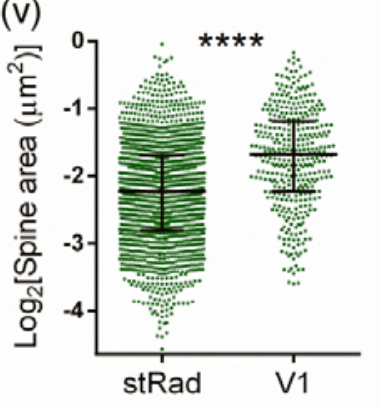

(iv)

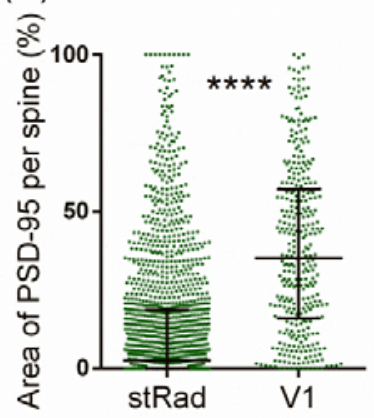

(vi)

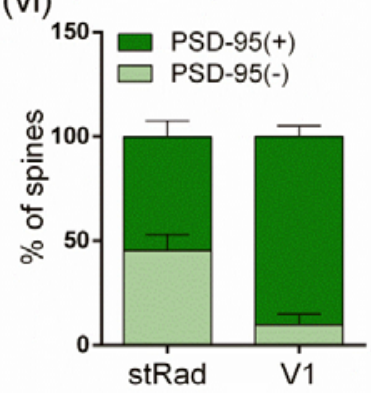

636

637

638

639

640

641

642

643

644

645

Figure 1-1. The comparison of PSD-95 protein expression and dendritic spines in the area

CA1 and visual cortex (V1). Representative high resolution images of PSD-95 immunostaining, dendritic fragments and co-localization of both in (i) stratum radiatum CA1 (stRad) and (ii) visual cortex (V1) of the control Thy1-GFP(M) mice, scale bars: $2 \mu \mathrm{m}$. (iii) There are more PSD-95 puncta per dendritic spine in V1, as compared to stRad (****p<0.0001, Mann-Whitney test). (iv) Total fraction of PSD-95 immunosignal per spine is higher in V1 than stRad $(* * * p<0.001$, MannWhitney test). (v) Dendritic spines in V1 are bigger than spines in stRad $(* * * * p<0.0001$, MannWhitney test). (iii-v) Each dot represents individual spine. (vi) There is higher frequency of spines with PSD-95 in V1 than in stRad. 


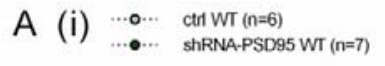

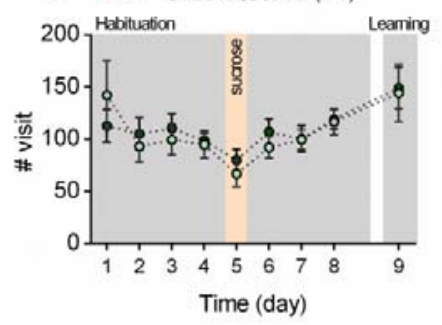

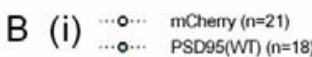

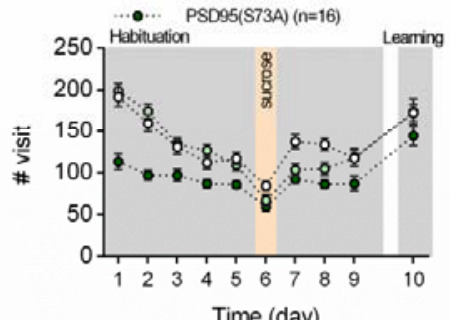

C (i) $\begin{array}{lll}\cdots \cdots & \cdots & \text { WT }(n=19) \\ \cdots & \cdots & \text { T286A (n=18) }\end{array}$

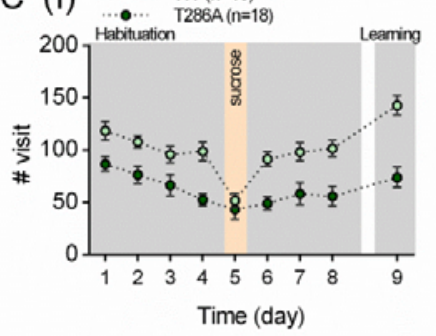

(ii)

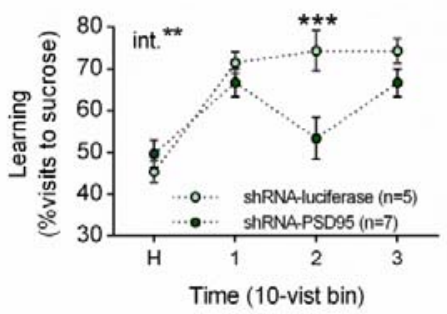

(ii)

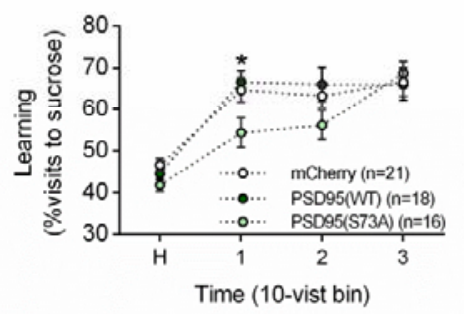

(ii)

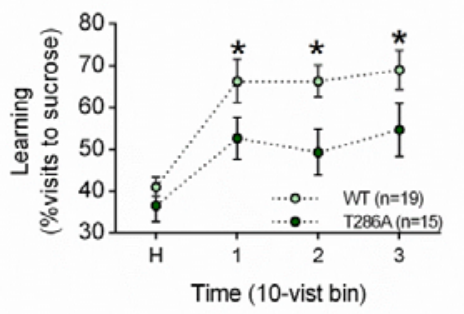

Figure 2-1. Mice activity during spatial training in the IntelliCages - the effect of modification

648

649

650

651

652

653

654

655

656

657

658

659

\section{of PSD-95 and CaMKII.}

(A) Mice behavior after downregulation of PSD-95 protein in the area CA1 by shRNA for PSD-95 in young mice. (i) shRNA for PSD-95 did not affect the general mice activity of the mice, measured as total number of visits in the corners (two-way ANOVA, shRNA: (i) virus: $\mathrm{F}_{1,99}=0.2544, \mathrm{p}=$ 0.615; time: $\mathrm{F}_{8,99}=3.579, \mathrm{p}=0.001$ ). (ii) shRNA did not affect preference for reward corner during learning measured as \% visit of sucrose in 10 -visit bins $(* * * \mathrm{p}<0.001$, two-way ANOVA with Sidak's multiple comparisons test, virus: $\mathrm{F}_{1,12}=3.536, \mathrm{p}=0.08$; time: $\mathrm{F}_{4,48}=10.93, \mathrm{p}<0.0001$; interaction: $\left.\mathrm{F}_{4,48}=3.950, \mathrm{p}=0.007\right)$. $\mathrm{H}$ - the last day of habituation for all experiments.

(B) Mice behavior after overexpression of PSD-95(WT) and (S73A) in CA1. (i) General mice activity in the experiment measured as total number of visits in the corners. Mice with PSD95(S73A) were less active than other groups during habituation but not during the training (RM ANOVA with Tukey's multiple comparisons test, virus: $\mathrm{F}_{2,691}=72.74, \mathrm{p}<0.0001$; time: $\mathrm{F}_{9,691}=$ 
660 36.81, $\mathrm{p}<0.0001$; interaction: $\mathrm{F}_{18,691}=3.127, \mathrm{p}<0.0001$ ). (ii) Preference for the reward corner

661 during learning measured as \% visits of sucrose in 10-visit bins $(* \mathrm{p}<0.05$, two-way ANOVA with

662 Sidak's multiple comparisons test, time: $\mathrm{F}_{4,260}=30.80, \mathrm{p}<0.0001$; virus: $\mathrm{F}_{2,260}=2.567, \mathrm{p}=0.078$ ).

663

664

665

666

667

668

669

670
(C) Young $\alpha$ CaMKII-T286A mutant mice. (i) General mice activity in the experiment measured as total number of visits in the corners. Mutants were less active than wild-type mice (RM ANOVA, genotype: $F_{1,283}=79.23, p<0.0001$, time: $F_{8,283}=6.591, p<0.0001$ ). (ii) Preference for reward corner during learning measured as \% visits to sucrose corner in 10-visit bins. Mutants had lower preference for the rewarded corner than wild-type mice $(* \mathrm{p}<0.05$, RM ANOVA with Tukey's multiple comparisons test, genotype: $F_{1,169}=12.91, p=0.0004$; time: $F_{4,169}=9.037, p<0.0001$ ). 

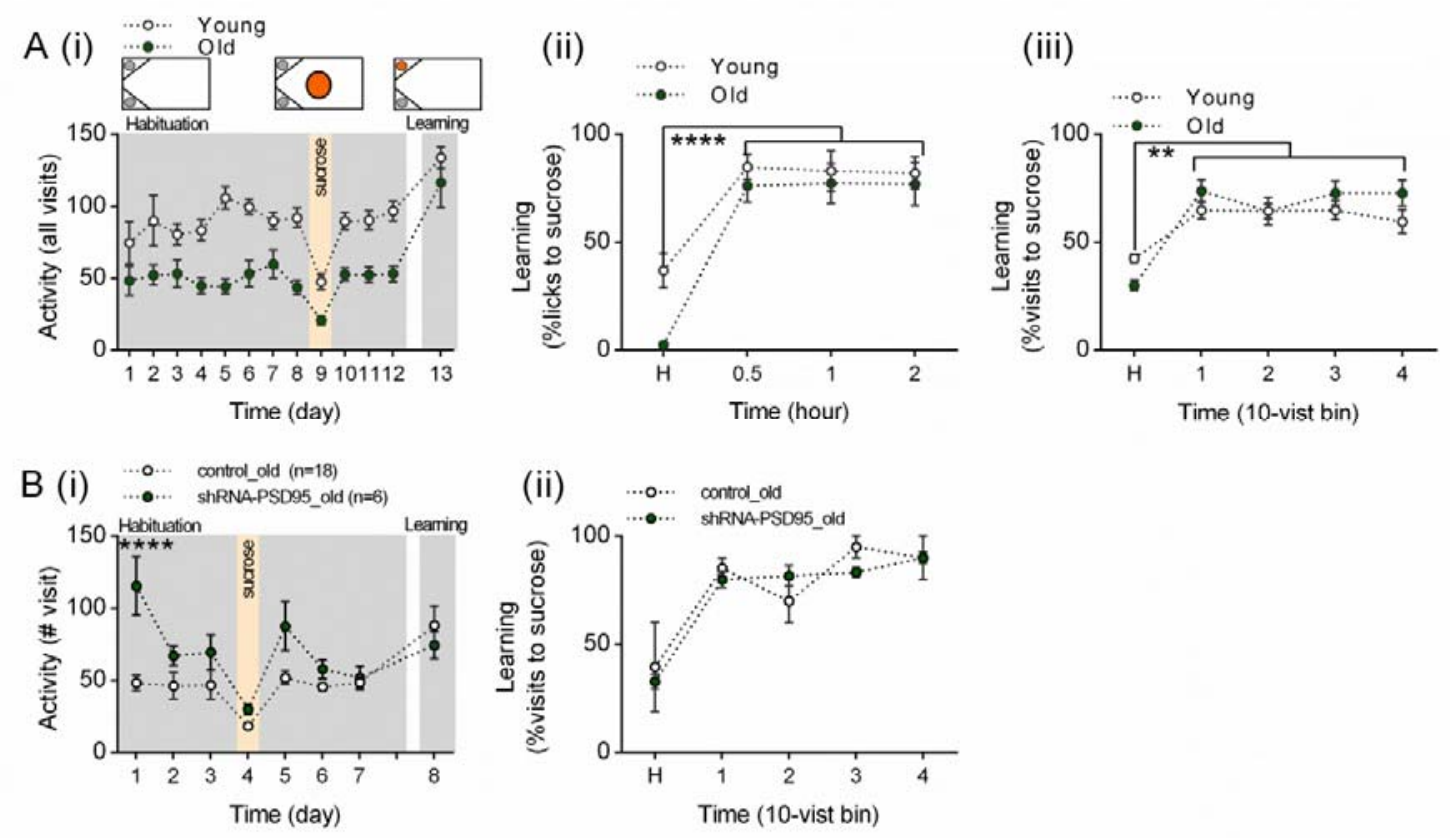

671

Figure 3-1. Old mice activity during reward-driven spatial learning in the IntelliCages - the

673

674

675

676

677

678

679

680

681

682

683

684

685

\section{effect of downregulation of PSD-95 in CA1.}

(A) (i) Top, cage setups during training. Gray circles, bottles with water; orange circles, bottles with sucrose. Bottom, total number of visits. Old mice performed less visits than young animals during the habituation phase, but not during spatial learning (RM ANOVA, age: $F_{2,593}=18.85, p<0.0001$; time: $F_{12,593}=25.65 \mathrm{p}<0.0001$; interaction: $\mathrm{F}_{24,593}=0.713, \mathrm{p}=0.840$ ). (ii) Mean \pm SEM preference for the reward corner during learning. Both young and old mice prefer to drink sucrose than water. $\mathrm{H}$, the last day of the habituation for all graphs $(* * * * \mathrm{p}<0.0001, \mathrm{RM}$ ANOVA with Sidak's multiple comparisons test, age: $\mathrm{F}_{1,211}=18.06, \mathrm{p}<0.0001$; time: $\mathrm{F}_{4,211}=38.29, \mathrm{p}<0,0001$; interactions: $\mathrm{F}_{4}$, $211=1.041, \mathrm{p}=0.387$ ). (ii) Preference for the reward corner during learning measured as $\%$ visits to sucrose corner in 10 -visit bins $(* * \mathrm{p}<0.01$, two-way ANOVA with Sidak's multiple comparisons test, time: $\mathrm{F}_{4,208}=18.36, \mathrm{p}<0.0001 ;$ age: $\left.\mathrm{F}_{1,208}=1.459, \mathrm{p}=0.2285\right)$.

(B) Old mice behavior after downregulation of PSD-95 protein in the area CA1 by shRNA for PSD95 in young mice. (i) shRNA-driven downregulation PSD-95 increased activity of old during habituation but not training $(* * * * \mathrm{p}<0.0001$, RM two-way ANOVA with Sidak's multiple comparisons test, shRNA: (i) virus: $\mathrm{F}_{1,19}=4.440, \mathrm{p}=0.0486$; time: $\mathrm{F}_{7,133}=12.3, \mathrm{p} \quad<\quad 0.0001$; 
688 interaction: $F_{7,133}=4.987, p<0.0001$ ). (ii) shRNA did not affect preference for reward corner

689 during learning measured as $\%$ visit of sucrose in 10 -visit bins $\quad(* * * p<0.001$, two-way ANOVA

690 with Sidak's multiple comparisons test, virus: $\mathrm{F}_{1,6}=0.1930, \mathrm{p}=0.6758$; time: $\mathrm{F}_{4,24}=39.68, \mathrm{p}<$

691 0.0001; interaction: $\left.\mathrm{F}_{4,24}=1.579, \mathrm{p}=0.2120\right) . \mathrm{H}-$ the last day of habituation for all experiments. 